\title{
Fundamental Concepts of Quantum Theories
}

\author{
Eliahu Comay \\ Charactell Ltd., Tel-Aviv, Israel \\ Email: elicomay@post.tau.ac.il
}

How to cite this paper: Comay, E. (2020) Fundamental Concepts of Quantum Theories. Open Access Library Journal, 7: e6970. https://doi.org/10.4236/oalib.1106970

Received: November 8, 2020

Accepted: December 5, 2020

Published: December 8, 2020

Copyright $\odot 2020$ by author(s) and Open Access Library Inc.

This work is licensed under the Creative Commons Attribution International License (CC BY 4.0).

http://creativecommons.org/licenses/by/4.0/

\section{(c) (i) Open Access}

\begin{abstract}
The foundations of the mathematical structure of quantum theories of a massive particle are the basis of this analysis. It proves the coherence of the particle-wave duality of quantum theories and the principle of complementarity as well. Furthermore, the noncommutativity of Hermitian operators proves that quantum theories are inherently indeterministic. This feature does not deny the fact that the classical limit of quantum theories agrees with classical physics. It is also shown that the foundations of the mathematical structure of quantum theories impose constraints on any specific quantum theory. It is proved that the first-order Dirac theory is consistent with all constraints. In contrast, second-order theories, such as the Klein-Gordon, the electroweak theory of the $W^{ \pm}$and the $Z$ particles, and the Higgs boson theory fail to do that. An analogous analysis proves that also the Majorana neutrino theory is inconsistent with fundamental requirements. Similarly, inconsistencies of Proca's idea about a massive photon are shown.
\end{abstract}

\section{Subject Areas}

Modern Physics

\section{Keywords}

Principles of Quantum Theories, Particles and Waves, Uncertainty and Determinism, Constraints on Acceptable Quantum Theories

\section{Introduction}

Intuition is an element that affects human activity, and the physical research is not free of it. However, it is now recognized that well established experimental data and the associated theories are essential elements of physics. The present work examines the mathematical structure of quantum theories of a massive particle and derives several conclusions. In particular, it proves that the particle-wave attributes of a quantum particle are compatible elements of the theory. 
In other words, the principle of complementarity is proved. Moreover, any problem with this issue stems from physically groundless human intuition.

The laws of Newtonian Mechanics (NM) say that the state of a mechanical system at a specific time in the future is accurately determined by solutions of an appropriate system of differential equations. Here a unique solution is obtained provided accurate values of the initial conditions are known. (The problem called stability of solutions of differential equations [1] is beyond the scope of this work). Therefore, the size of inaccuracy stems from the current limitation of experimental devices. This situation improves with the progress of time. The plain meaning of this matter is that, in principle, the concept of determinism holds for cases where NM holds. However, one cannot be sure that determinism must hold for systems that NM fails to explain. A well-known example of this failure is a system of a proton and an electron. Here classical physics says that the electron accelerates, and the system emits electromagnetic radiation (see [2], pp. 175-176; [3], p. 657). This result is inconsistent with the stability of the ground state of the hydrogen atom. It can be concluded that it is not a priori clear whether the validity of the concept of determinism holds for systems where NM fails, like the ground state of the hydrogen atom. Indeed, it is proved below that the mathematical framework of quantum theories is a logically coherent structure that explains many effects that NM cannot explain. It turns out that quantum theories are incompatible with the concept of determinism.

Further analysis of the elements of quantum theories proves that constraints are imposed on specific quantum theories that describe the behavior of elementary massive particles. The compatibility between these constraints and the Dirac equation is demonstrated. In contrast, it is proved that problems exist with the Klein-Gordon (KG) theory, the electroweak theory of the $W^{ \pm}$and the $Z$ particles, the theory of the Higgs particle, the Majorana theory of the neutrino, and Proca's idea of a massive photon.

In summary, the paper proves these issues: the principle of complementarity of the particle-wave concepts; the inherent indeterminism of quantum theories and its compatibility with the deterministic feature of NM; the meaning of specific constraints that are imposed on any given quantum theory.

Units where $\hbar=c=1$ are used. Greek indices run from 0 to 3. Most formulas take the standard form of a relativistic covariant expression. The metric is diagonal and its entries are $(1,-1,-1,-1)$. An upper dot denotes the time-derivative. In the above-mentioned units, there is just one kind of dimension, and an appropriate power of the length $\left[L^{n}\right]$ denotes the dimension of a physical quantity. The second section describes the principles used in the analysis. The third section presents elements of quantum theories. The fourth section discusses conclusions that are derived from the issues that are mentioned in the previous sections. The last section summarizes this work.

\section{Principles Used in the Analysis}

Every physical theory must satisfy two general requirements: It must have a co- 
herent mathematical structure and it must adequately describe relevant experimental data. Physics is already a mature science and several principles are regarded as correct elements that every theory must abide with. These principles yield some requirements that are used in this work.

R.1) An acceptable physical theory is not expected to explain every phenomenon but only the data that belongs to its domain of validity. For example, NM adequately explains cases where the particles' velocity is much smaller than the speed of light, and quantum effects can be ignored. These limitations determine the validity domain of NM. This example illustrates a general principle where any given theory should have a domain of validity.

If the domain of validity of theory $T_{B}$ contains all cases that are included in the domain of validity of theory $T_{A}$ then there are correspondence relationships between these theories. Here $T_{B}$ takes a higher hierarchical position with respect to $T_{A}$. For example, in the case of relativistic classical mechanics (RCM) there is no limitation on the particles' velocity. Hence, the domain of validity of this theory includes that of NM, because it also explains cases of small velocities that can be explained by NM. It means that RCM takes a higher hierarchical status with respect to NM. However, the meaning of a lower rank theory should not be underestimated because it imposes requirements on the higher rank theory: An appropriate limit of expressions of the higher rank theory should agree with corresponding expressions of the lower rank theory. For example, the relativistic expression for the energy of a moving particle is

$$
\mathcal{E}=\frac{m}{\sqrt{1-v^{2}}}
$$

where $m$ denotes the particle's mass and $v$ is its velocity. If $v \ll 1$ then (1) takes the form

$$
\mathcal{E}=m+m v^{2} / 2
$$

(see [2], p. 27). The second term of (2) is the NM expression for the kinetic energy of a moving particle.

This work uses the correspondence between Quantum Field Theory (QFT), Relativistic Quantum Mechanics (RQM), Quantum Mechanics (QM), and NM. The correspondence between QM and NM is proved in many textbooks. For example, "classical mechanics must therefore be a limiting case of quantum mechanics." (see [4], p. 84; [5], pp. 25-27, 137, 138). The correspondence between QFT and QM is stated in a well-known textbook: "First, some good news: quantum field theory is based on the same quantum mechanics that was invented by Schroedinger, Heisenberg, Pauli, Born, and others in 1925-26, and has been used ever since in atomic, molecular, nuclear and condensed matter physics" (see [6], p. 49).

Figure 1 illustrates the relationships between the above mentioned 4 theories. Indeed, contrary to NM, QM explains data of microscopic effects like electronic states of atoms, etc. The relativistic covariant structure of RQM explains data 


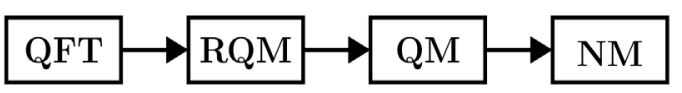

Figure 1. Hierarchical relationships between 4 theories. (See text. The meaning of the acronyms is explained in the second paragraph of the introduction and in the paragraph that begins below (2)).

that QM cannot explain. QFT explains data that RQM cannot explain. For example, experiments prove that the proton contains three valence quarks and the probability of additional quark-antiquark pairs [7] [8] [9] [10]. Hence, the problem of the proton structure is outside the RQM domain of validity, because $\mathrm{RQM}$ applies to systems where the number of particles is well-defined.

Another example of the correspondence between physical theories connects RQM and RCM. Figure 2 illustrates these relationships.

R.2) The variational principle is regarded as a fundamental element of the structure of a quantum theory. Here the variation applies to the action

$$
S(\psi)=\int \mathrm{d}^{4} x \mathcal{L}(\psi, \psi, \mu),
$$

where $\psi$ is a quantum function and $\mathcal{L}\left(\psi, \psi_{, \mu}\right)$ is a Lagrangian density. The symbol $\psi$ is a shorthand notation of $\psi(x)$, where $x$ denotes the four space-time coordinates. For example, a well-known textbook supports this approach and states: "all field theories used in current theories of elementary particles have Lagrangians of this form" (see [6], p. 300). Here the action $S$ and the Lagrangian density $\mathcal{L}$ are mathematically real Lorentz scalars. These attributes are used below.

This form of the Lagrangian density does not explicitly depend on the space-time coordinates. Hence, the Noether theorem proves that the theory conserves energy, momentum, and angular momentum (see [11], pp. 17-19). This result provides strong support for using this form of the variational principle as a fundamental element of a quantum theory.

R.3) The de Broglie principle states that a quantum particle has wave properties, and the wavelength of a free quantum particle is related to its momentum. Thus, the phase of such a wave is

$$
\Phi=\mathrm{e}^{i(\boldsymbol{k} \cdot \boldsymbol{x}-\omega t)} .
$$

Here $\boldsymbol{k}$ takes the value of the particle's momentum $\boldsymbol{p}$, and $\omega$ is its energy (see [4], pp. 119, 120; [5], p. 3).

\section{Elements of the Structure of a Quantum Theory}

Let us begin with the Dirac theory of an electrically charged elementary particle. The Lagrangian density of this particle is

$$
\mathcal{L}_{D}=\bar{\psi}\left[\gamma^{\mu}\left(i \partial_{\mu}-e A_{\mu}\right)-m\right] \psi,
$$

where the relation $\bar{\psi}=\psi^{\dagger} \gamma^{0}$ is used, $\gamma^{\mu}$ are the standard Dirac matrices (see [12], p. 17), and $A^{\mu}=(V, \boldsymbol{A})$ denote the electromagnetic 4-potential (see [11], p. 84, [13], p. 78). The term that contains $A_{\mu}$ is the interaction term of a charged Dirac particle. 


\section{$\mathrm{QFT} \rightarrow \mathrm{RQM} \rightarrow \mathrm{RCM}$}

Figure 2. Hierarchical relationships between 3 theories. (see text. The meaning of the acronyms is explained in the paragraph that begins above (1) and below (2)).

The Hamiltonian density is a significant element of the theory. The Legendre transformation of (5) yields the Dirac Hamiltonian density

$$
\mathcal{H}_{D}=\pi \dot{\psi}-\mathcal{L}=\psi^{\dagger}[\boldsymbol{\alpha} \cdot(-i \nabla-e \boldsymbol{A})+\beta m+e \phi] \psi,
$$

where $\alpha, \beta$ are the ordinary Dirac matrices (see [11], p. 87). Here $\psi$ is the generalized coordinate and $i \psi^{\dagger}$ is its conjugate momentum (see [13], p. 52). The product $\psi^{\dagger} \psi$ of (6) is the Dirac density (see [12], p. 9), and the quantities inside the square brackets compose the Dirac Hamiltonian operator (see [12], p. 11).

An important property of the Dirac Hamiltonian density (6) is that

$$
\psi^{\dagger} \psi=|\psi|^{2} \geq 0
$$

It means that the functions $\psi$ can be used for the construction of a Hilbert space (see [6], pp. 49, 50). This space indicates that the Dirac theory yields mathematically real quantities provided operators like $\psi^{\dagger} \hat{O} \psi$ of this Hilbert space are Hermitian. It is proved in Section 2 that the action should be a mathematically real quantity. In the case of the Dirac theory, this requirement is satisfied if the Hamiltonian density (6) is Hermitian. This issue is regarded as the standard structure of quantum theories (see [6], pp. 49, 50; [14], pp. 144-150).

It is proved in textbooks that a solution of a free Dirac particle takes the required form of the de Broglie wave (4) (see e.g. [12], p. 28).

Let us examine the correspondence between quantum theories and NM. In $\mathrm{NM}$, the energy of a closed system is a well-defined quantity. Therefore, the correspondence principle 1 means that quantum theories must have an expression for energy and that the appropriate limit of this expression should agree with the NM expression for energy. The Dirac Hamiltonian of (6) satisfies this requirement. Indeed, the non-relativistic limit of the Dirac equation agrees with the Schroedinger equation [15], and the classical limit of the Schroedinger equation corresponds to NM (see [4], p. 84; [5], pp. 25-27, 137, 138). Hence, the Dirac Hamiltonian is an essential element of the theory, because it shows the compatibility of this theory with the required correspondence between quantum theories and NM, which is illustrated in Figure 1.

\section{Discussion}

This section shows that the concepts described in Sections 2, 3 yield coherent quantum theory as well as constraints that apply to specific versions of QFT.

A) The particle-wave duality.

PW.1) Landau and Lifshitz prove that in RCM an elementary particle is pointlike: "In other words, within the framework of classical theory elementary particles must be treated as points." (see [2], pp. 46, 47). 
The correspondence between QFT and RCM, which is illustrated in Figure 2 entails that the quantum theories of an elementary particle should also have this attribute. It turns out that the general form of the Lagrangian density (3) describes a pointlike particle. Indeed, $\psi(x)$ of (3) depends on the four space-time coordinates. Hence, it can describe the probability of finding the particle at $x$, but it cannot describe how the structure of a finite particle is distributed at the vicinity of $x$, namely at points $x+\delta x$. For example, the $\pi^{+}$meson is not an elementary particle, but a $u \bar{d}$ quark-antiquark bound state (see [16], p. 40). Hence, its quantum function takes the form $\psi\left(\boldsymbol{x}_{1}, \boldsymbol{x}_{2}, t\right)$, where $\boldsymbol{x}_{1}, \boldsymbol{x}_{2}$ denote the $u \bar{d}$ coordinates, respectively. Evidently, $\psi(x)$ depends on four independent variables, whereas $\psi\left(\boldsymbol{x}_{1}, \boldsymbol{x}_{2}, t\right)$ depends on seven independent variables.

These theoretical results have very strong experimental support. For example, the electron is an elementary Dirac particle, and experiments find a very small upper bound of its size $r_{e}<10^{-20} \mathrm{~cm}$ [17]. On the other hand, the $\pi^{+}$charge radius is $r_{\pi^{+}}=0.66 \mathrm{fm}$ [18]. This value is larger than the upper bound of the electron's radius by 7 orders of magnitudes.

PW.2) Let us examine the ground state of the hydrogen atom. Here the Dirac equation "was validated by accounting for the fine details of the hydrogen spectrum in a completely rigorous way" [15]. The calculation of the hydrogen atom ground state energy takes this general form

$$
E=\int \psi^{\dagger} \hat{H} \psi \mathrm{d}^{3} r
$$

where $\psi=\psi(x, y, z)$ is the ground state wave function and $\hat{H}$ denotes all terms of the Hamiltonian (6). The interaction energy depends on the electromagnetic potential. This quantity is stronger at points where the electron-proton distance is short and weaker for points where this distance is long. Expression (8) means that it regards a situation where the electron exists simultaneously at all points of the integration region. This kind of calculation is a vital element of the theory because it proves energy conservations of transitions between atomic states.

Conclusion: The success of the Dirac theory of the electron and the integral (8) mean that in a quantum theory, the electron simultaneously exists at all points of the spatial region where the integral is calculated. This property pertains to the wave features of a quantum particle.

PW.3) Item PW.1 shows that a quantum particle is pointlike. On the other hand, item PW.2 shows that at any given instant, a quantum particle is spread over a spatial region, which means that it has wave-like attributes. Here is an explanation of the logical coexistence of these concepts.

Examples of classical waves, like a wave in a pond or a sound wave, are a manifestation of the motion of elements of a medium. Here the system's energy is distributed throughout a quite large spatial region of the medium. Hence, people who adhere to concepts of classical physics may regard the pointlike and the wave properties of a quantum particle as a contradiction. However, this conclusion is unjustified. Indeed, the scientific foundation of the wave phenomenon re- 
lies not on its human interpretation but on the mathematical structure of the wave equation. In the case of constant velocity $v$, the form of this equation is

$$
\nabla^{2} \phi=\frac{1}{v^{2}} \frac{\partial^{2} \phi}{\partial t^{2}}
$$

(see [19], p. 5). This equation depends on the velocity $v$, and it is independent of any medium. In classical physics, properties of the medium determine $v$, and in a quantum theory, $v$ is determined by the de Broglie expression (4). Furthermore, it is shown above that the quantum function $\psi(x)$ describes a pointlike particle, and it satisfies the wave equation as well. These arguments explain why the particle-wave properties of a quantum particle are consistent elements of quantum theories.

This outcome has strong experimental support. Take for example the double-slit interference experiment [20]. Here an appropriate beam of electrons (or another kind of a quantum particle) is split into two sub-beams that pass through two slits and produce an interference pattern on a screen. Each electron behaves as a wave that exists at the two spatially distinct regions of the sub-beams. Later, the interference pattern is generated as an assembly of distinct dots, where each of which results from a single electron that hits the screen. Here the interference pattern describes the wave property, and each dot describes the particle property.

A classical wave depends on a medium by means of which the wave phenomenon is manifested. Here the energy of the process is distributed throughout an appropriate portion of the medium. This effect looks contradictory to the particle concept. However, it is explained above why the independence of the wave equation (9) on the existence of a medium enables reconciliation between the particle and the wave notions of quantum theories. This conclusion is mentioned in textbooks. For example: "Bohr, who was guided by the empirical fact of the dual nature of matter, has elevated this program to a principle of complementarity. According to this principle, wave and particle nature are considered complementary aspects of matter, both equally essential for a full description of the phenomena" (see [14], p. 7). It is shown above that the medium-independence of the wave equation (9) proves the principle of complementarity.

B) The quantum uncertainty principle.

UNC.1) It is explained in Section 3 why the Hermitian operator of the Hamiltonian represents the system's energy. The correspondence principle which is depicted in Figure 1 means that the appropriate limit of other quantities of a quantum theory should agree with corresponding quantities that are determined by classical physics. These quantities take mathematically real values. Hence, like in the Hamiltonian's case, the value of a dynamical variable of a quantum particle should be derived from a Hermitian operator (see [6], pp. 49, 50).

A mathematical property of Hermitian operators is that not every two Hermitian operators commute. As an example, let us examine the commutation relations of the $x$ coordinate and its conjugate momentum. These relations and the 
associated uncertainty are

$$
x p_{x}-p_{x} x=i \rightarrow \Delta x \Delta p_{x} \geq 1 .
$$

(see [4], p. 87). These relations are well documented in textbooks: "The Heisenberg uncertainty relation is thus seen to be a direct consequence of the noncommutativity of the position and the momentum operators" (see [14], p. 160).

The theory's relativistic covariant form yields the corresponding energy-time uncertainty relations, where the Hamiltonian $H$ is the energy operator. Here the Hamiltonian and the time operators do not commute, because $i \partial / \partial t=H$. Hence, one obtains

$$
H t-t H=i \rightarrow \Delta E \Delta t \geq 1 \text {. }
$$

UNC.2) The uncertainty relations between quantum operators means that the determinism of classical physics does not hold in quantum theories. The following argument proves the indeterministic aspect of quantum theories.

Consider a classical theory of a system of $n$ elementary particles that relies on a given Lagrangian

$$
L\left(q_{i}, \dot{q}_{i}, t\right)
$$

(see e.g. [21], p. 35; [22], p. 2). This Lagrangian depends on the particles' coordinates, their time-derivatives, and the time. The Legendre transformation casts this Lagrangian into the Hamiltonian formalism, where the theory is derived from

$$
H\left(q_{i}, p_{i}, t\right)
$$

(see e.g. [21], p. 337; [22], p. 131). This Hamiltonian depends on the coordinates, their conjugate momenta, and the time. The theory based on the Lagrangian (12) is equivalent to the theory that is based on the Hamiltonian (13).

In the case of a closed system, the deterministic attribute of classical physics says that if the initial values of the coordinates and the momenta are accurately given at time $t_{0}$ then the theory provides accurate values of the state of the system at any given time $t$.

Evidently, this goal cannot be achieved in quantum mechanics, simply because the coordinate-momentum uncertainty relation (11) prevents an accurate determination of these variables at any given instant. It means that quantum theories should be base on different principles, and they cannot follow the deterministic solutions of (13). However, the correspondence principle that is described in Section 2 proves the logical compatibility of quantum theories with NM.

UNC.3) Experiments provide convincing results of the validity of the energy-time uncertainty relations (11). Thus, it is explained in a textbook that a well-defined energy "conforms with the uncertainty relation $\Delta E \Delta t>\hbar$, which implies that a quantum state with a precise energy $(\Delta E=0)$ is possible only if there is unlimited time available to determine that energy. Stationary states are of such a nature in view of the constancy of $|\psi|$ in time" (see [14], p. 46). 
Data of the proton and the $\Delta(1232)$ baryon illustrate this issue. Thus, the proton is a stable particle and the experimental uncertainty of its mass is $\Delta m<6 \mathrm{eV}$ [18]. This quantity reflects an inaccuracy that stems from the limitations of the present experimental technology. In principle, the accuracy of measurements of the proton mass improves with the progress of time. In contrast, the $\Delta^{++}(1232)$ baryon decays rapidly due to a strong interaction process. The energy-width of the graph that depicts its creation in a $\pi^{+} p$ collision is about $117 \mathrm{MeV}$ (see [16], p. 134 and [18]). This energy-width stems from the energy-time uncertainty relation, and it is larger than the proton's value by 7 orders of magnitude. A quite large width is also found for other particles whose decay is a strong interaction process, like the $\rho(770)$ and the $\omega(782)$ mesons, etc. (see [18]).

Conclusion: appropriate experiments systematically demonstrate the validity of the uncertainty principle.

C) Here are some other conclusions that are derived from the principles of Sections 2, 3.

RES.1) The energy uncertainty property of a quantum particle explains the classically forbidden effect, where a particle tunnels through a potential barrier that is higher than its energy (see [14], pp. 74, 75). Well-known effects, like the nuclear $\alpha$ decay, the nuclear fusion, and modern electronic devices are based on this phenomenon [23].

RES.2) The Schroedinger QM theory and the correspondence principle that is depicted in Figure 1 impose strong restrictions on the acceptability of quantum theories. Thus, QM proves that the probability of a quantum particle is

$$
\rho=\psi^{*} \psi \rightarrow \int \psi^{*} \psi \mathrm{d}^{3} x=1,
$$

where $\psi$ is a normalized function (see [14], p. 37). It means that the dimension of the Schroedinger function $\psi$ is $\left[L^{-3 / 2}\right]$.

Dimension is a discrete quantity and it does not change in a limit process. Hence, the correspondence principle says that a quantum function that is treated by QFT should also have this dimension. In the units used herein $\hbar=c=1$, and the action is dimensionless. Hence, the dimension of the QFT Lagrangian density (3) is $\left[L^{-4}\right]$. The Dirac Lagrangian density (5) has a term $m \bar{\psi} \psi$. Mass and energy have the dimension $\left[L^{-1}\right]$. It follows that the dimension of the Dirac function $\psi$ is $\left[L^{-3 / 2}\right]$. This value is consistent with the value of the Schroedinger function, which is derived from (14). It means that the Dirac theory satisfies this aspect of the correspondence principle. In contrast, a Lagrangian density that contains a term like $m^{2} \phi^{\dagger} \phi$ proves that the dimension of the quantum function $\phi$ is $\left[L^{-1}\right]$. This is a violation of the correspondence principle. This argument proves that the theories of the KG particle (see [13], p. 16), the electroweak theory of the $W^{ \pm}$and the $Z$ particles (see [24], p. 518), and that of the Higgs boson (see [13], p. 715), are inherently wrong. The same is true with the Proca's theory of a massive photon (see e.g. [3], pp. 597-601). The recent data of the upper bound of the photon's mass is $m<10^{-18} \mathrm{eV}$ [18]. This upper bound is 
smaller than the electronic mass by a factor of about 24 orders of magnitudes. Therefore, experiments refute Proca's idea about a massive photon.

RES.3) The Schroedinger theory takes a mathematically complex form. Hence, the correspondence principle says that theories of a massive particle that take a mathematically real form are unacceptable. This argument applies to the real KG particle, the $Z$ boson, the Higgs boson, the Majorana neutrino, and the Proca's idea of a massive photon.

\section{Summary}

The correspondence principle, the variational principle, and the de Broglie particle-wave relations are used for the construction of quantum theories of a massive particle. It is proved that the point-like attribute of an elementary quantum particle is compatible with its wave attribute. In other words, the principle of complementarity is proved. It is also clarified why the mathematical property of noncommutativity of Hermitian operators, like those of the position and the momentum, is the reason for the intrinsic indeterminism of quantum theories. It is also explained why this issue is consistent with the deterministic concept of classical mechanics.

The correspondence between QFT and the Schroedinger nonrelativistic theory (see [6], p. 300) imposes constraints on the mathematical structure of any specific QFT of a massive particle. A proof shows that the first-order Dirac's theory of a spin-1/2 massive particle meets these requirements. In contrast, second-order quantum theories, like the KG theory, the electroweak theory of the $W^{ \pm}$and the $Z$ particles, the Higgs boson theory, and the Majorana theory of the neutrino, are inconsistent with the above mentioned basic requirements. The paper proves the inconsistency of Proca's idea of a massive photon. The mathematically complex form of the Schroedinger theory yields analogous results.

\section{Conflicts of Interest}

The author declares no conflicts of interest regarding the publication of this paper.

\section{References}

[1] Stability Theory. https://en.wikipedia.org/wiki/Stability_theory

[2] Landau, L.D. and Lifshitz, E.M. (2005) The Classical Theory of Fields. Elsevier, Amsterdam.

[3] Jackson, J.D. (1975) Classical Electrodynamics. John Wiley, New York.

[4] Dirac, P.A.M. (1958) The Principles of Quantum Mechanics. Oxford University Press, London. https://doi.org/10.1063/1.3062610

[5] Schiff, L.I. (1955) Quantum Mechanics. McGraw-Hill, New York.

[6] Weinberg, S. (1995) The Quantum Theory of Fields. Vol. I. Cambridge University Press, Cambridge. https://doi.org/10.1017/CBO9781139644167

[7] ATLAS Collaboration (2012) Determination of the Strange Quark Density of the 
Proton from ATLAS Measurements of the $\mathrm{W} \rightarrow \mathrm{lv}$ and $\mathrm{Z} \rightarrow 1 \mathrm{l}$ Cross Sections. Physical Review Letters, 109, Article ID: 012001. https://arxiv.org/abs/1203.4051

[8] Alberg, M. (2008) Parton Distributions in Hadrons. Progress in Particle and Nuclear Physics, 61, 140-146. https://doi.org/10.1016/j.ppnp.2007.12.003

[9] Reimer, P.E. and the Fermilab SeaQuest Collaboration (2016) Sea Quarks in the Proton. EPJ Web of Conferences, 113, Article No. 05012.

https://www.epj-conferences.org/articles/epjconf/pdf/2016/08/epjconf_fb2016_0501 2.pdf https://doi.org/10.1051/epjconf/201611305012

[10] Reimer, P.E. (2020) Measurement of the Flavor Asymmetry in the Proton's Sea Quarks.

https://www.anl.gov/event/measurement-of-the-flavor-asymmetry-in-the-protons-s ea-quarks

[11] Bjorken, J.D. and Drell, S.D. (1965) Relativistic Quantum Fields. McGraw-Hill, New York. https://doi.org/10.1063/1.3047288

[12] Bjorken, J.D. and Drell, S.D. (1964) Relativistic Quantum Mechanics. McGraw-Hill, New York

[13] Peskin, M.E. and Schroeder, D.V. (1995) An Introduction to Quantum Field Theory. Addison-Wesley, Reading.

[14] Merzbacher, E. (1970) Quantum Mechanics. John Wiley, New York.

[15] Dirac Equation. https://en.wikipedia.org/wiki/Dirac_equation

[16] Griffiths, D. (2008) Introduction to Elementary Particles. 2nd Edition, Wiley-VCH, Weinheim.

[17] Dehmelt, H. (1988) A Single Atomic Particle Forever Floating at Rest in Free Space: New Value for Electron Radius. Physica Scripta, 1988, 102. https://doi.org/10.1088/0031-8949/1988/T22/016

[18] Zyla, P.A., et al. (2020) Progress of Theoretical and Experimental Physics, 2020, 083C01. https://pdg.lbl.gov/2020/listings/contents_listings.html

[19] Coulson, C.A. (1961) Waves. Oliver and Boyd, Edinburgh.

[20] Double-Slit Experiment. https://en.wikipedia.org/wiki/Double-slit_experiment

[21] Goldstein, H., Poole, C. and Safko, J. (2002) Classical Mechanics. 3rd Edition, Addison Wesley, San Francisco. https://doi.org/10.1119/1.1484149

[22] Landau, L.D. and Lifshitz, E.M. (1960) Mechanics. Pergamon, Oxford.

[23] Quantum Tunneling. https://en.wikipedia.org/wiki/Quantum_tunnelling

[24] Sterman, G. (1993) An Introduction to Quantum Field Theory. Cambridge University Press, Cambridge. https://doi.org/10.1017/CBO9780511622618 